\title{
Hipersensibilidad a corticoides y manejo en asma severa. A propósito de un caso
}

\author{
MARÍA IGNACIA DURÁN W.*, VÍCTOR PINOCHET F.*, MIGUEL AGUAYO C.*, \\ CLAUDIA AZÓCAR B.* y JORGE YÁÑEZ V.*
}

\section{Hipersensitivity to corticosteroids and management of severe bronchial asthma. Case study}

Hypersensitivity reactions to corticosteroids are rare in the general population, they fall into two categories: 'immediate', typically mediated by immunoglobulin E (IgE), which includes anaphylaxis after administration of a drug in a short period of time. Its reported prevalence is $0.3-0.5 \%$. Another reaction is 'not immediate', which manifests itself in a time longer than one hour after the administration of the drug. We reviewed the literature with the aim of improving and clarifying the treatment in asthmatic patients with this condition. It was found that the possible routes to generate these reactions are intranasal, aerosol by inhaler, oral and parenteral. Facing this condition requires a close and detailed evaluation of the clinical history, symptoms and side reactions to the suspected drug. Finally, when choosing which corticosteroid to use, the patient's safety is paramount, and control of the disease is also essential.

Key words: Anaphylaxis; Asthma; Immunoglobulin E; Adrenal cortex hormones; Nebulizers and vaporizers.

\section{Resumen}

Las reacciones de hipersensibilidad a corticoides son raras en la población general, se dividen en dos categorías: Inmediatas, típicamente mediadas por Inmunoglobulina E (IgE), donde se incluye la anafilaxia luego de la administración de un fármaco en un corto período. Su prevalencia descrita es de 0,3-0,5\%. Otra reacción es la 'no inmediata', que se manifiesta en un tiempo mayor de una hora después de la administración del fármaco. Se revisó la literatura con el objetivo de mejorar y aclarar el tratamiento en pacientes asmáticos que poseen esta condición. Se encontró que las vías posibles para generar estas reacciones son intranasal, aerosol por inhalador, oral y parenteral. Frente a esta condición se requiere una evaluación estrecha y detallada de la historia clínica, síntomas y reacciones secundarias al fármaco sospechoso. Finalmente, al momento de elegir tipo de corticoide a usar es primordial la seguridad del paciente logrando, además, el control de la enfermedad.

Palabras clave: Anafilaxia; Asma; Inmunoglobulina E; Hormonas córtico-adrenales; nebulizadores y vaporizadores.

\section{Introducción}

Los corticoides, han sido de gran utilidad desde los años 40s', su uso en diversas patologías médicas determinado por su efecto antiinflamatorio e inmunodepresor, sus variadas formulaciones químicas y vías de administración han generado un alto impacto en el uso de estos. Desde su inicio la utilidad se ha limitado sobre la base de la documentación de efectos adversos diversos $\mathrm{y}$ en ocasiones graves, por lo cual sus beneficios se han contrapesado con sus potenciales efectos secundarios, siendo estos mecanismos aun no conocidos de forma completa ni tampoco el efecto en los diversos sistemas.

Los efectos adversos son variados, si bien

* Servicio de Medicina Interna, Hospital Clínico Regional de Concepción y Departamento de Medicina Interna, Facultad de Medicina, Universidad de Concepción. Concepción, Chile. 
es paradójico pensar que ocurran reacciones de hipersensibilidad, estas sí pueden estar presentes como manifestaciones de tipo locales y sistémicas.

Ya en la década de 1950, Kendall informó de 20 reacciones sistémicas que ocurrieron en una cohorte de 2.256 pacientes que habían recibido un total de 6.700 inyecciones de corticosteroides ${ }^{1}$. Dulloo y cols revisaron a 213 niños tratados con corticoesteroides (CS) intravenosos para diversas condiciones reumatológicas. Su estudio calculó que la tasa de anafilaxia era del $0,5 \%{ }^{2}$. Un estudio más reciente ha situado la prevalencia estimada de hipersensibilidad a los esteroides después de la administración sistémica en $0,3 \%{ }^{3}$.

\section{Descripción del caso}

Mujer de 66 años, hipertensa con asma bronquial severa hace 6 años, usuaria de teofilina, montelukast, loratadina, salbutamol y bromuro de ipratropio. Alergia conocida a hidrocortisona, penicilina y aspirina. Sin antecedente de tabaquismo.

Hace 6 años inicia sintomatología caracterizada por tos, sibilancias y disnea principalmente secundaria al uso de aspirina. En una de las exacerbaciones de asma presentó dos episodios de paro cardiorrespiratorio posterior a inyección de hidrocortisona. Posterior a estos eventos no se logra controlar su asma bronquial y la paciente mantiene sintomatología respiratoria variable según las estaciones del año.

Dentro de los exámenes durante este período destaca la espirometría de 2015 (Figura 1).

Radiografía tórax sin hallazgos significativos, eosinófilos $510 / \mathrm{mm}^{3}$ e IgE $39,3 \mathrm{mg} / \mathrm{L}$.

La paciente tras persistir con asma severa no controlada, consulta nuevamente en Urgencia Hospital Guillermo Grant Benavente en marzo de 2017 por cuadro de disnea de 2 días de evolución, sibilancias audibles y uso de musculatura accesoria, destacaba en el examen físico taquicardia 132 ciclos por min, frecuencia respiratoria 35 ciclos por min, temperatura $36,5{ }^{\circ} \mathrm{C}$, oximetría $95 \%$.
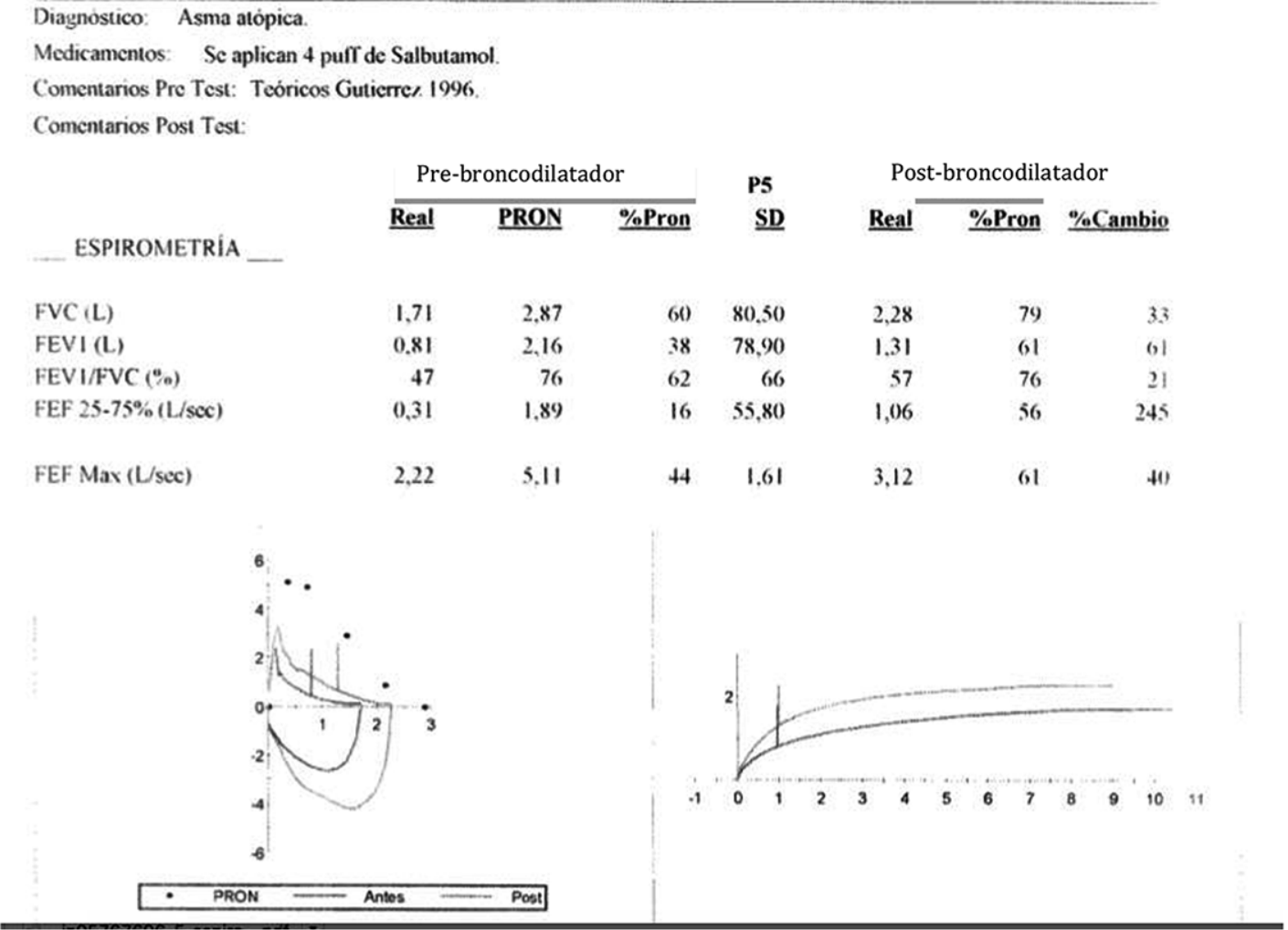

Imagen de sistema Galileo Hospital Guillermo Grant Benavente

Figura 1. Espirometría de 2015. Alteración ventilatoria obstructiva severa con CVF disminuido que se modifica significativamente con broncodilatador. Pron: valor teórico normal. 
Exámenes de ingreso: gasometría arterial $\mathrm{pH} 7,40$ $\mathrm{PO}_{2}: 79 \mathrm{mmHg}, \mathrm{PCO}_{2}: 40 \mathrm{mmHg}$ Bicarbonato: $24 \mathrm{mEq} / \mathrm{L}$ y saturación de la hemoglobina con $\mathrm{O}_{2}$ $96 \%$, ácido láctico $3,0 \mathrm{mmol} / \mathrm{L}$; hemograma predominio de eosinófilos $590 / \mathrm{mm}^{3}$ y función renal normal. Se realiza nebulizaciones con salbutamol y Berodual, se indica sulfato de magnesio y aminofilina en bomba de infusión continua. Dada su mala evolución con insuficiencia respiratoria aguda asociado a deficiente mecánica ventilatoria y compromiso de conciencia se decide ingreso a UCIM, intubar y conectar a ventilación mecánica invasiva. La gasometría arterial al inicio de la ventilación mecánica mostró $\mathrm{pH} 7,24 \mathrm{PO}_{2}$ $219 \mathrm{mmHg}, \mathrm{PCO}_{2} 61 \mathrm{mmHg}$. Bicarbonato 26,1 $\mathrm{mEq} / \mathrm{L}$, saturación de la hemoglobina $100 \%$, dentro de estudio etiológico resulta con IFI (inmunofluorescencia indirecta) de virus respiratorios negativa y radiografía de tórax que muestra hiperinflación pulmonar sin foco de condensación.

Ingresa a la Unidad de Paciente Crítico y se agrega Ketamina como manejo de su status asmático sin iniciar corticoides dentro de los primeros 3 días de ingreso, debido al antecedente de paro cardiorrespiratorio secundario al uso de este fármaco.

Para evaluar tolerancia y prueba terapéutica con corticoides de menor riesgo de reacción cruzada se realiza test de parche con Dexametasona subcutánea $0,5 \mathrm{mg}$, el cual resulta sin complicaciones, y se decide inicio de tratamiento con dosis plena y por horario $4 \mathrm{mg}$ cada $8 \mathrm{~h}$ endovenosa, lo cual resultó un éxito. Dado la buena respuesta y tras la revisión en la literatura, se decide iniciar administración de Deflazacort $30 \mathrm{mg}$ día vía oral por 7 días posterior a extubación, sin que la paciente presentara ningún tipo de reacción adversa a este medicamento.

Tras persistir buena evolución y con la intención de proporcionar un mejor manejo y control de la enfermedad se realiza prueba terapéutica con inhalador que incluyó a corticoide de grupo $\mathrm{D} 1$, Beclometasona dipropionato $50 \mu \mathrm{g} / \mathrm{Salbu}-$ tamol sulfato $120 \mu \mathrm{g}$ en dosis de 2 puffs cada 6 h. Luego de iniciar manejo con este inhalador se logró objetivar una disminución considerable de sibilancias, tos diurna y disnea.

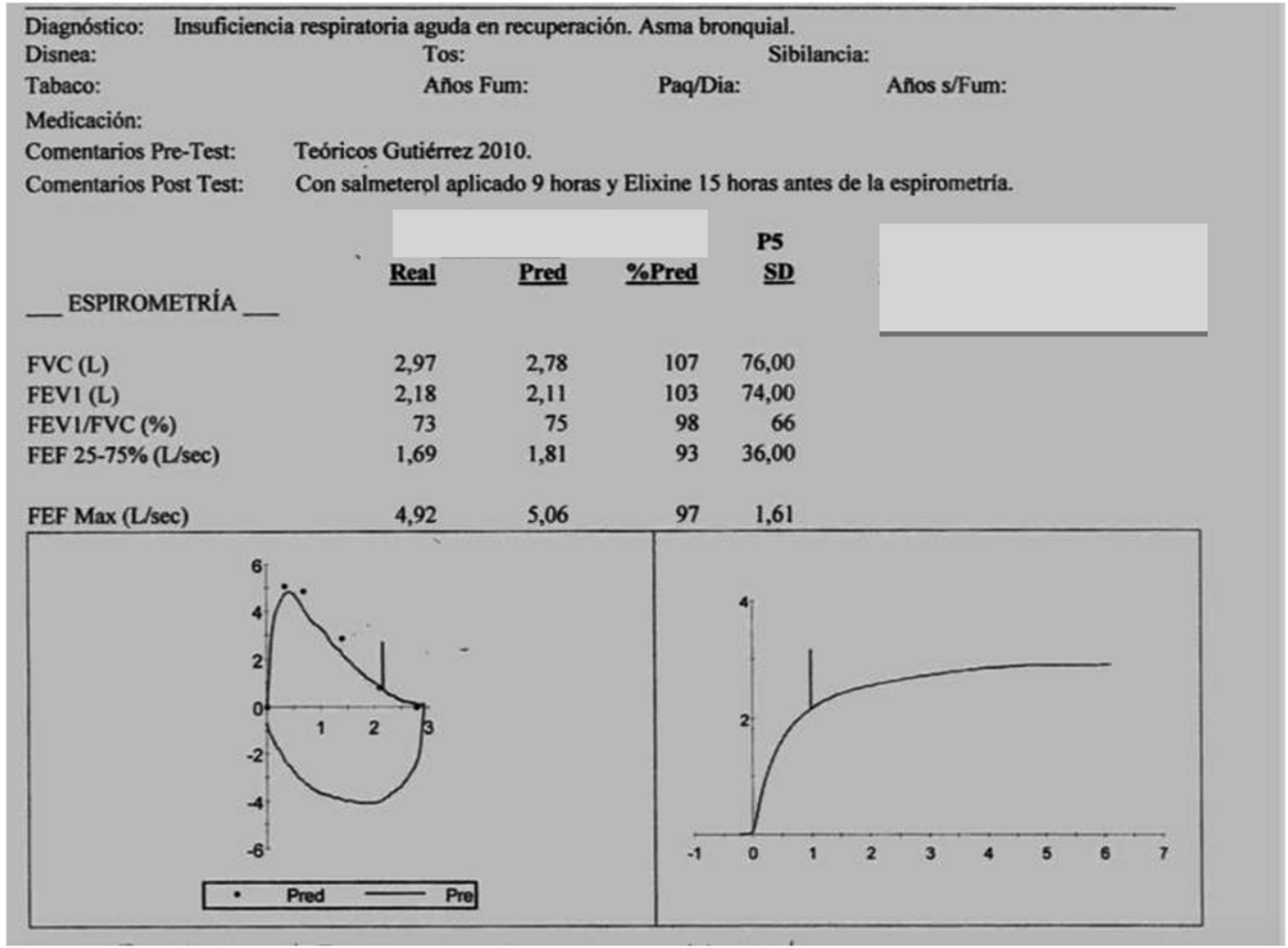

Imagen de sistema Galileo Hospital Guillermo Grant Benavente

Figura 2. Espirometría dentro de límites normales. Pred: valor teórico normal. 
Siete días después del nuevo tratamiento se realiza una nueva espirometría (Figura 2) la cual resulta totalmente normal. La paciente actualmente se mantiene asintomática, disnea mMRC 1 y sin ningún tipo de reacción adversa secundaria al tratamiento con corticoide inhalado.

\section{Discusión y Comentarios}

Sabemos que el asma es un trastorno inflamatorio crónico de las vías respiratorias inferiores, con una distribución mundial que afecta a todas las edades ${ }^{11,12}$. La prevalencia del asma está aumentando en todo el mundo. Se estima que por lo menos 300 millones de personas tienen actualmente asma, y se espera que más de 100 millones se habrán agregado antes de $2025^{12,13}$. A pesar de los progresos en el diagnóstico y tratamiento del asma, esta sigue siendo una de las enfermedades más subdiagnosticadas y subtratadas, dando como resultado altas tasas de morbilidad y discapacidad.

La inflamación de los bronquios es la principal causa subyacente de la hiperreactividad de las vías respiratorias y de la broncoconstricción. Los CS son la piedra angular del manejo del asma, ya que son los agentes antiinflamatorios más potentes actualmente disponibles. Los corticoesteroides inhalados (ICS) son recomendados, como terapia de primera línea a dosis bajas para el asma persistente leve y como terapia preferida a dosis medias o en combinación con un agonista $\beta-2$ de acción prolongada para Asma persistente moderada ${ }^{13,14}$. La terapia combinada con altas dosis de ICS se recomienda sólo para pacientes con asma persistente grave ${ }^{15,16}$.

Los ICS se prefieren a la administración oral, ya que se han asociado con un número significativamente menor de efectos secundarios. Los efectos secundarios sistémicos con las dosis convencionales de ICS son insignificantes, pero se necesita tener precaución en relación a los efectos secundarios sistémicos y locales cuando se utilizan dosis más altas.

El broncoespasmo causado por CS sistémicos es más frecuente en la población de asmáticos con alergia a la aspirina. Se ha propuesto que la aspirina produciría leucotrienos broncoconstrictores desviando el metabolismo del ácido araquidónico de la vía de la ciclooxigenasa a la 5- lipooxigenasa. Ciertos CS pueden inhibir la liberación de ácido araquidónico, privando a los asmáticos alérgicos a la aspirina de las prostaglandinas necesarias para mantener la geometría normal de la vía aérea. El mecanismo del bronco- espasmo inducido por CS es desconocido pero un mecanismo propuesto sería el mencionado efecto inhibitorio sobre la ciclo-oxigenasa ${ }^{17,20}$.

El empeoramiento de los síntomas con el tratamiento CS no siempre significa fallo del tratamiento y debe sospecharse alergia a los mismos. El diagnóstico se confirma por una clínica sugestiva tras la exposición, la presencia de $\operatorname{IgE}$ específica o pruebas cutáneas o de provocación positivas $^{17}$.

\section{Patogenia}

Existen dos tipos de hipersensibilidad, la primera es la 'hipersensibilidad inmediata' mediada por IgE que ocurre durante la primera hora de administración del fármaco, y la 'hipersensibilidad retardada' mediada por linfocitos $\mathrm{T}$, que es la más común y ocurre después de una hora de uso y principalmente luego de aplicación de productos tópicos. La reacción de hipersensibilidad tipo inmediata, aunque es menos común se asocia principalmente al uso de corticoides sistémicos, generando anafilaxia debido a la liberación masiva de agentes inflamatorios de mastocitos y basófilos, esto en el peor de los escenarios.

El cortisol por ser de bajo peso molecular y alta lipofilia es capaz de penetrar fácilmente los tejidos, resultando como producto de degradación de la posición C21, para formar Glioxal esteroide, el que se une a arginina y forma un hapteno que se comporta como agente inmunogénico.

El CS sintético administrado en grandes dosis puede haptenizar con estas proteínas séricas circulantes, lo que a su vez generará alergenos inmunogénicos estables. Los pacientes con riesgo particular incluyen aquellos con asma, hipersensibilidad al ácido acetilsalicílico u otros fármacos antiinflamatorios no esteroideos y antecedentes de nefritis o trasplante renal ${ }^{4}$.

\section{Clasificación de corticoides}

El sistema de clasificación de Coopman (o ABCD), basado en la publicación de 1989 sobre la alergia a esteroides de Coopman y cols., los divide en cuatro grupos compuestos de reacción cruzada $^{5}$. Esta clasificación fue validada adicionalmente por Goossens y cols., basándose en los resultados de los ensayos de parches cutáneos obtenidos de una gran cohorte de pacientes alérgicos a CS (Tabla 1$)^{6}$.

Estos datos sobre la verdadera reactividad alérgica cruzada entre CS tópica se proporcionan 
Tabla 1. Clasificación ABCD de los corticoesteroides ${ }^{5}$

\begin{tabular}{|c|c|c|c|c|c|}
\hline Clasificación & A & B & C & D1 & D2 \\
\hline Características & $\begin{array}{l}\text { Sin sustitución en } \\
\text { el anillo D excepto } \\
\text { ester o tioester de } \\
\text { cadena corta en C } 21\end{array}$ & Acetónidos & $\begin{array}{l}\text { Metilación en } \\
\text { C16 }\end{array}$ & $\begin{array}{l}\text { Metilación en } \\
\text { C16 sustitución } \\
\text { halogénica }\end{array}$ & $\begin{array}{l}\text { Sin metilación en } \\
\text { C16 y sin sustitución } \\
\text { halogénica. Cadena } \\
\text { ester lateral en C17 y } \\
\text { posiblemente en C } 21\end{array}$ \\
\hline Ejemplos & $\begin{array}{l}\text { Hidrocortisona } \\
\text { Prednisolona } \\
\text { Metilprednisolona } \\
\text { Cortisona acetato } \\
\text { Prednisona } \\
\text { Tixocortol pivalato* }\end{array}$ & $\begin{array}{l}\text { Triamcinolona } \\
\text { Fluocinolona } \\
\text { Halcilonida } \\
\text { Desonida } \\
\text { Fluocinonida } \\
\text { Budesonida }\end{array}$ & $\begin{array}{l}\text { Betametasona } \\
\text { Dexametasona } \\
\text { Desoximetasona } \\
\text { Fluocortolona }\end{array}$ & $\begin{array}{l}\text { Clobetasona } \\
\text { Clobetasol } \\
\text { Beclometasona } \\
\text { Betametasona }\end{array}$ & $\begin{array}{l}\text { Hidrocortisona- } \\
\text { 17-propionato o } \\
\text {-butirato } \\
\text { Metilprednisolona } \\
\text { aceponato } \\
\text { Prednicarbato } \\
\text { Difluprednato }\end{array}$ \\
\hline
\end{tabular}

*Usado en los ensayos de parches cutáneos (patch tests).

mediante pruebas de parche con compuestos a los que los pacientes sensibles nunca han sido previamente expuestos.

La reactividad cruzada de los compuestos de CS tiene consecuencias prácticas para la identificación de los CS más frecuentemente responsables de la sensibilización, así como la elección de qué agente utilizar para las pruebas de diagnóstico. En general, las reacciones más positivas se han observado en el grupo A, grupo $\mathrm{B}$, y en aquellos compuestos pertenecientes al subgrupo D2.

Este último grupo a su vez muestra una tasa significativa de reactividad cruzada entre sus propios miembros, así como a los compuestos del grupo A y budesonida (grupo B). Por otro lado, el grupo $\mathrm{C}$ y los del subgrupo D1 producen muy pocas reacciones de contacto alérgico y, además, los compuestos del grupo $\mathrm{C}$ y del grupo D1 exhiben una reactividad cruzada mínima con otros grupos esteroideos.

Nakamura y cols., realizaron un estudio sobre siete pacientes adultos con asma, cada uno de los cuales tenía una historia de reacción anafiláctica sistémica después de la administración intravenosa de CS para el tratamiento del asma aguda.

Las reacciones anafilácticas sistémicas a los CS intravenosos ocurrieron en asmáticos severamente atópicos con antecedentes de exposición previa a corticoides parenterales, independientemente de edad y sexo.

En los siete sujetos, se indujeron reacciones anafilácticas después de la administración intravenosa de preparaciones de CS que contenían succinato, es decir, hidrocortisona y metilprednisolona. La administración de corticosteroides que contienen fosfato, es decir, dexametasona y betametasona, era segura y dio como resultado una mejora de la reacción anafiláctica. El examen inmunológico con pruebas cutáneas sugirió que estas reacciones anafilácticas eran efectivamente mediadas por $\operatorname{Ig} \mathrm{E}^{7}$.

\section{Presentación clínica de la hipersensibilidad}

Existe una variada presentación clínica de los efectos a corticoides, dependiendo de la vía de administración, dosis, estructura química y cantidad del fármaco. Estas reacciones pueden ir desde anafilaxia como hipersensibilidad tipo I a manifestaciones tópicas por vía de hipersensibilidad tipo IV. Otras reacciones incluyen: morfea, dermatitis sistémica de contacto, erupción máculo-papular exantemática, dermatitis exfoliativa, síndrome de Stevens-Johnson, eritroderma generalizado, así como pustulosis exantemática generalizada aguda.

Con respecto a las manifestaciones respiratorias, el broncoespasmo inducido por corticosteroides es más frecuente en los asmáticos con antecedentes de alergia a la aspirina ${ }^{8-10}$. En casos de empeoramiento del asma a pesar del tratamiento adecuado, se debe considerar el broncoespasmo inducido por corticosteroides. Las tasas de reacciones de hipersensibilidad son también más altas en pacientes que reciben dosis crónicas o recurrentes de terapia tópica o sistémica, especialmente parenteral administrada con esteroides ${ }^{10}$.

\section{Tipos de vías alergénicas}

Importante es conocer las distintas vías, en primer lugar, la vía tópica, ya que por su amplio uso ha generado mayor hipersensibilidad, siendo más común la reacción provocada por 
los productos del grupo A, como budesonida e hidrococortisona.

Dentro de la vía sistémica se encuentra prednisona y prednisolona como más frecuentes, generando reacciones como anafilaxia, urticaria generalizada, angioedema, dolor abdominal, sibilancias y hasta paro cardiorrespiratorio.

En lo que se refiere a la vía inhalatoria, la posibilidad de reacciones alérgicas no parece clara, siendo budesonida la que genera mayor reacción alérgica y principalmente lesiones cutáneas, como eczema en nariz, ojos, boca, cuello y cara, además de disfagia, eritema en vías respiratorias, y edema provocando su obstrucción total.

Es llamativo que los pacientes asmáticos son menos propensos a presentar episodios de alergia a los glucocorticoides por vía inhalatoria, esto puede deberse a falta de documentación de los casos, pero también se podría explicar por el desarrollo de tolerancia en estos enfermos. La importancia de presentar reacciones alérgicas en estos pacientes es la gravedad y mayor intensidad en los afectados.

\section{Uso de agentes alternativos}

La administración de corticoides como tratamiento de primera línea en una variedad de condiciones supone un verdadero desafío para el médico tratante.

A pesar de su uso generalizado, la alergia al CS sigue siendo rara vez reportada. Esto podría explicarse debido a que es una condición poco común, o estamos subdiagnosticando esta reacción. La incidencia exacta de hipersensibilidad CS es difícil de estimar y se limita a informes de casos y pequeños estudios clínicos.

La alergia CS no suele ser a un fármaco específico. Por lo tanto, un paciente sensibilizado a un agente corre el riesgo de presentar reacciones de hipersensibilidad a otro CS debido a co-sensibilización o reactividad cruzada de estos compuestos, especialmente si son miembros de la misma familia. Todas las vías de sensibilización, incluyendo la cutánea, pueden conducir a reacciones sistémicas.

Aunque es raro, la hipersensibilidad a CS puede tener consecuencias perjudiciales para el paciente y afectar adversamente su manejo, lo que provoca mala calidad de vida, limitación de las opciones de tratamiento, aumento de la morbilidad e incluso mortalidad debido al regular control de la enfermedad. Se debe considerar la dermatitis alérgica de contacto con esteroides cuando los síntomas cutáneos empeoran a pesar de la terapia con CS tópica. Dado que la administración de CS inhalados y sistémica puede causar reacciones hipersensibles, es razonable incluir la hipersensibilidad a CS en el diagnóstico diferencial de un paciente asmático cuyos síntomas empeoran después del inicio de la terapia. Sin embargo, puede ser difícil y frustrante para el clínico distinguir entre una reacción de hipersensibilidad y el deterioro de la enfermedad inflamatoria para la cual se prescribió la terapia con CS. Este desafío de diagnóstico enfatiza la importancia de una evaluación exhaustiva para identificar el fármaco culpable y encontrar una alternativa segura para el paciente. La elección de un agente esteroide alternativo no sólo es primordial para la seguridad del paciente, sino que también mejora la preocupación de desarrollar una reacción de hipersensibilidad alérgica, y potencialmente mortal, de esteroides. Esta evaluación se vuelve particularmente significativa en pacientes de alto riesgo donde los CS son un tratamiento que puede salvar la vida.

\section{Conclusión}

La administración de CS sigue siendo la piedra angular del tratamiento en enfermedades alérgicas e inflamatorias. Paradójicamente, los CS pueden desencadenar una reacción de hipersensibilidad con una amplia variedad de mecanismos fisiopatológicos, no siendo ellos del todo conocidos.

A pesar de su uso generalizado, la alergia a CS sigue siendo rara vez reportada. Si esto es porque es realmente una condición poco común, o está bajo diagnóstico y por lo tanto no reconocido, no está claro.

La clasificación de ABCD con subclasificación D1, D2 es útil para dirigir la búsqueda de un agente de reemplazo, pero no puede reemplazar una evaluación sistemática e individualizada del perfil de sensibilización y tolerancia de cada paciente. Existen corticosteroides más nuevos, como el Deflazacort, que no pertenece en el sistema de clasificación $\mathrm{A}, \mathrm{B}, \mathrm{C}, \mathrm{D}$, pero que demostró ser útil en el manejo de exacerbación de Asma sin ningún efecto adverso descrito. Además, es importante considerar la menor respuesta alérgica que presentan los CS que pertenecen al grupo D1, ya que tampoco existió reacción alérgica con este tipo de ICS que era lo que faltaba en nuestra paciente como gran pilar de tratamiento. Debemos destacar que la modificación y mejoría de la función pulmonar con ICS es relevante, demostrando en un corto plazo que los ICS son la piedra angular del tratamiento ${ }^{18}$.

Es importante considerar que, ante el uso de $\mathrm{CS}$, los clínicos, con independencia de la vía de 
administración, deben valorar la posibilidad de que se produzcan reacciones alérgicas potencialmente graves; sería de ayuda la confirmación diagnóstica utilizando test cutáneo, y se debe tener precaución a la hora de administrar un nuevo corticoide si ello fuera necesario, mayor aun en pacientes con historia de alergias conocidas.

Con respecto al caso clínico descrito, es relevante tener en consideración la gran respuesta al grupo de corticoides con menos reacción alérgica descrita, siendo muy interesante e importante la buena respuesta de manejo y control del Asma Bronquial que continúa presentando la paciente en el último tiempo. Es de utilidad conocer los distintos tipos de grupos de corticoides y así tener la información adecuada en caso de tener que usarlos, como fue en este caso.

\section{Bibliografía}

1.- KENDALL PH. Untoward effects following local hydrocortisone injection. Ann Phys Med. 1958; 4: 170-5.

2.- DULLOO AR, RUDDY FH, CONGEDO TV, SEIDEL JG, MCILWAIN ME. Experimental verification of modeling results for a PGNAA system for nondestructive assay of RCRA metals in drums. Appl Radiat Isot 2000; 53: 499-505.

3.- BORJA JM, GALINDO PA, FEO F, GÓMEZ E. Urticaria to methylprednisolone sodium hemisuccinate. Allergy 2001; 56: 791.

4.- VENTURA MT, CALOGIURI GF, MURATORE L, DI LEO E, BUQUICCHIO R, FERRANNINI A, et al. Cross -reactivity in cell- mediated and IgE-mediated hypersensitivity to glucocorticoids. Curr Pharm Des 2006; 12: 3383-91.

5.- COOPMAN S, DEGREEF H, DOOMS-GOOSSENS

A. Identification of cross-reaction patterns in allergic contact dermatitis from topical corticosteroids. $\mathrm{Br} \mathrm{J}$ Dermatol 1989; 121: 27-34.

6.- GOOSSENS A, MATURA M, DEGREEF H. Reactions to corticosteroids: some new aspects regarding crosssensitivity. Cutis 2000; 65: 43-5.

7.- NAKAMURA H, MATSUSE H, OBASE Y, MITSUTA $\mathrm{K}$, TOMARI S, SAEKI S, et al. Clinical evaluation of anaphylactic reactions to intravenous corticosteroids in adult asthmatics. Respiration 2002; 69: 309-13.

8.- $\quad$ SHETH A, REDDYMASU S, JACKSON R. Worsening of asthma with systemic corticosteroids. A case report and review of literature. J Gen Intern Med 2006; 21 : C11-C13.

9.- POLOSA R, PROSPERINI G, PINTALDI L, REY JP, COLOMBRITA R. Anaphylaxis after prednisone. Allergy 1998; 53: 330-1.

10.- LAINE-CESSAC P, MOSHINALY H, GOUELLO JP,
GESLIN P, ALLAIN P. Severe anaphylactoid reactions after intravenous cortico- steroids. Report of a case and review of the literature. Therapie 1990; 45: 505-8.

11.- WORLD ALLERGY ORGANIZATION (WAO). White Book on Allergy, 2011-2012. Disponible en: http:// www.worldallergy.org/UserFiles/file/ExecSummary -2013-v6-hires.pdf (Fecha de consulta: Septiembre de 2016).

12.- AKINBAMI L, MOORMAN J, BAILEY C, et al. Trends in Asthma Prevalence, Health Care Use, and Mortality in the United States, 2001-2010. NCHS Data Brief, No. 94, 2012. Disponible en: http://www.cdc. gov/nchs/data/ databriefs/db94.pdf (Fecha de consulta: Septiembre de 2016).

13.- MASOLI M, FABIAN D, HOLT S, et al. Global burden of asthma. 2004. Disponible en: https://www.ncbi.nlm. nih.gov/pubmed/15080825 (Fecha de consulta: 25 de octubre de 2009).

14.- MASOLI M, FABIAN D, HOLT S, BEASLEY R. The global burden of asthma: executive summary of the GINA Dissemination Committee report. Allergy 2004; 59: 469-78.

15.- SMITH MJ, HODSON ME. High dose beclomethasone inhaler in the treatment of asthma. Lancet 1983; 1: 2659.

16.- TARLO SM, DAVIES GM, MINTZ S. Six month double blind, controlled trial of high dose concentrated beclomethasone dipropionate in the treatment of severe chronic asthma. Chest 1988; 93: 998-1002.

17.- KLEINERT MM, RHODIUS E, COMIGNANI P. [An almost fatal asthma crisis induced by glucocorticoids. Sometimes it happens]. Medicina (Buenos Aires) 2007; 67 (6 pt2): 772-4.

18.- FERRAZ AMARO I, DÍAZ GONZÁLEZ F, GONZÁLEZ T. Alergia a los glucocorticoides: ¿una paradoja? Med Clin (Barc) 2003; 120: 141-5.

19.- VATTI RR, ALI F, TEUBER S, CHANG C, GERSHWIN ME. Hypersensitivity Reactions to Corticosteroids. Clin Rev Allergy Immunol 2014; 47: 26-37.

20.- BAECK M, MAROT L, NICOLAS JF, PILETTE C, TENNSTEDT D, GOOSSENS A. Allergic hypersensitivity to topical and systemic corticosteroids: a review. Allergy 2009; 64: 978-94.

Correspondencia a:

Dra. M. Ignacia Durán W.

Servicio de Medicina, Facultad de Medicina,

Universidad de Concepción.

Email: mi_duran86@hotmail.com 